\title{
Cobertura do tratamento diretamente observado (DOTS) da Tuberculose no Estado de São Paulo (1998 a 2004)
}

\author{
COVERAGE OF DIRECTLY OBSERVED TREATMENT (DOTS) OF TUBERCULOSIS \\ IN THE STATE OF SÃO PAULO (1998-2004)
}

\author{
COBERTURA DEL TRATAMIENTO DIRECTAMENTE OBSERVADO (DOTS) \\ DE LA TUBERCULOSIS EN EL ESTADO DE SÃO PAULO (1998 - 2004).
}

\begin{abstract}
Tereza Cristina Scatena Villa1, Elisangela Gisele de Assis², Mayra Fernanda Oliveira ${ }^{3}$, Ricardo Alexandre Arcêncio ${ }^{4}$, Roxana Isabel Cardozo Gonzales ${ }^{5}$, Pedro Fredemir Palha ${ }^{6}$
\end{abstract}

\section{RESUMO}

Objetivando descrever a cobertura do DOTS, analisou-se o Tratamento Supervisionado (TS) nos 36 municípios prioritários para o controle da TB do Estado de São Paulo entre 1998 e 2004. Estudo exploratório fundamentado no Banco de Dados EPI-TB da Secretaria de Estado da Saúde de São Paulo. Foi elaborado um instrumento de coleta de dados e calculada a cobertura do TS. Os dados foram dispostos no programa Excel. Nos municípios que implantaram o TS em 1998, a cobertura foi inferior a $20 \%$. Em 1999, metade dos municípios alcançou cobertura entre 11 e $49 \%$. Em 2000 , houve $100 \%$ de implantação do TS, porém, o valor máximo de cobertura alcançado foi $61 \%$. Entre 2001 e 2002, a cobertura aumentou em $69,44 \%$ dos municípios. Em 2003, em 22 municípios a cobertura estava abaixo de 50\%. Em 2004 houve tendência de aumento de cobertura em $63,89 \%$ destes municípios.

\section{DESCRITORES}

Tuberculose.

Vigilância.

Atenção primária à saúde.

Serviços de Saúde.

\begin{abstract}
The Supervised Treatment (ST) in the 36 priority municipalities for TB control of the State of São Paulo between 1998 and 2004 was analyzed with the aim of describing the coverage of the DOTS. This exploratory study used information from the State of São Paulo's Health Secretary's EPI-TB database. An instrument of data collection was elaborated and the coverage of the ST was calculated. The data was put in the Excel program. In the municipalities that implemented the ST in 1998, coverage was under $20 \%$. In 1999 half of the municipalities had coverage between $11 \%$ and $49 \%$. In 2000 , the implementation was $100 \%$, but the maximum coverage was $61 \%$. Between 2001 and 2002 the coverage increased in $69.44 \%$ of the municipalities. In 2003, coverage was below of $50 \%$ in 22 municipalities. In 2004 there was a trend to increasing coverage in $63.89 \%$ of the municipalities.
\end{abstract}

\section{KEY WORDS}

Tuberculosis.

Surveillance.

Primary health care.

Health Services.

\begin{abstract}
RESUMEN
Teniendo como objetivo describir la cobertura del DOTS se analizó el Tratamiento Supervisado (TS) en los 36 municipios prioritarios para el control de la TB del Estado de Sao Paulo entre 1998 y 2004. Se trata de un estudio exploratorio fundamentado en el Banco de datos EPI-TB de la Secretaría de Estado de la Salud de São Paulo. Fue elaborado un instrumento de recolección de datos y calculada la cobertura del TS. Los datos fueron digitados en el programa Excel. En los municipios con TS en 1998 la cobertura fue inferior al 20\%. En 1999 , la mitad de los municipios alcanzaron una cobertura entre 11 y $49 \%$. En el 2000 hubo el $100 \%$ de implantación del TS, sin embargo la cobertura máxima alcanzada fue del $61 \%$. Entre 2001 y 2002, la cobertura aumentó del $69,44 \%$ de los municipios. En el 2003, en 22 municipios la cobertura estaba abajo del 50\%. En el 2004 tenía tendencia al aumento de la cobertura en el $63.89 \%$ de estos municipios.
\end{abstract}

\section{DESCRIPTORES \\ Tuberculosis. \\ Vigilancia. \\ Atención primaria de salud. \\ Servicios de Salud.}

\footnotetext{
${ }^{1}$ Coordenadora da Área de Pesquisa Operacional da Rede TB na Escola de Enfermagem de Ribeirão Preto, Universidade de São Paulo (EERP-USP). Ribeirão Preto, SP, Brasil. tite@eerp.usp.br ${ }^{2}$ Graduanda do 6은 Semestre de Enfermagem da Escola de Enfermagem de Ribeirão Preto, Universidade de São Paulo (EERP-USP). Bolsista de Iniciação Científica da FAPESP. Ribeirão Preto, SP, Brasil. elisasis@eerp.usp.br ${ }^{3}$ Doutoranda da Escola de Enfermagem de Ribeirão Preto, Universidade de São Paulo (EERP-USP). Ribeirão Preto, SP, Brasil. mayrafo@eerp.usp.br ${ }^{4}$ Doutorando da Escola de Enfermagem de Ribeirão Preto, Universidade de São Paulo (EERP-USP). Ribeirão Preto, SP, Brasil. ricardo@eerp.usp.br ${ }^{5}$ Doutora em Enfermagem pela Escola de Enfermagem, Universidade de São Paulo (EERP-USP). Ribeirão Preto, SP, Brasil. roxanacardozoandre@yahoo.com.br ${ }^{6}$ Professor Doutor do Departamento de Enfermagem Materno-Infantil em Saúde Pública da Escola de Enfermagem de Ribeirão Preto, Universidade de São Paulo (EERP-USP). Ribeirão Preto, SP, Brasil. palha@eerp.usp.br
} 


\section{INTRODUÇÃO}

A Tuberculose (TB) é uma endemia que esteve presente como problema de saúde pública no Brasil durante todo o século $X X^{(1)}$, e ficou conhecida como a calamidade negligenciada $^{(2)}$. Ela esteve controlada até meados da década de 80 , sendo então esquecida pelas políticas públicas de saúde, ressurgindo no final desta década com grande número de casos. A partir de 1993, a Organização Mundial da Saúde (OMS) declarou a TB como emergência mundial|(3).

Segundo estimativa da OMS, um terço da população mundial está infectada com a TB sendo que de dois biIhões de infectados, oito milhões desenvolverão a doença e 1,8 milhões morrerão dela a cada ano ${ }^{(4)}$. Existem nove milhões de casos novos de TB e aproximadamente dois milhões de mortos pela TB em 2004 ${ }^{(5)}$.

O Brasil tem ocupado a 16a posição entre os 22 países responsáveis por $80 \%$ do total de casos de TB no mundo ${ }^{(5)}$. Com 179.108.134 habitantes ${ }^{(6)}$, a TB é a 9a causa de internações por doenças infecciosas, ocupa o $7^{\circ}$ lugar em gastos com internações pelo Sistema Único de Saúde (SUS), além de ocupar a 4a posição como causa de mortalidade por doenças infecciosas. Na capital do Estado de São Paulo no ano de 2004 o número de casos chega a 7197, estando acima da média nacional de mortalidade por TB que é de aproximadamente 3,2 óbitos por 100.000 habitantes $^{(7)}$.

Para o combate da TB foi proposta a estratégia DOTS (Directly Observed Treatment Short Course) pela OMS em 1993 , no intuito de atingir $85 \%$ de sucesso de tratamento e $70 \%$ de detecção de $\operatorname{casos}^{(8)}$.

Esta estratégia é composta por cinco elementos: compromisso dos governos ao suporte financeiro das atividades de controle da TB; deteç̧ão de casos pela baciloscopia de escarro entre pacientes sintomáticos que se apresentam espontaneamente ao serviço de saúde; suprimento regular de todos os medicamentos essenciais antituberculose; sistema padronizado de registro e notificação que permita conclusões seguras sobre os resultados do tratamento para cada paciente e do controle do programa de forma geral; e por último, regime de tratamento padronizado de seis a oito meses para todos os casos confirmados a partir de testes positivos de secreção, com tratamento supervisionado (TS) pelo menos nos dois meses iniciais ${ }^{(5)}$.

Em 1998, ocorre a implantação do Plano Nacional de Controle da Tuberculose (PNCT) no Brasil o qual

define a TB como prioridade entre as políticas governamentais de saúde, estabelece diretrizes para ações e fixa metas para o alcance de seus objetivos ${ }^{(3)}$.

Desde a implantação do DOTS no Brasil, a taxa de cobertura do TS ou tratamento Diretamente Observado
$(\mathrm{DOT})^{(9)}$, vem aumentando no período entre 1998 a 2004 (3\%, 7\%, 7\%, 32\%, 25\%, 33,6\% e 52\%), respectivamente, sem alcançar a meta preconizada pela $\mathrm{OMS}^{(5)}$.

\section{Esta estratégia}

aumenta as taxas de cura acima de $95 \%$ até mesmo nos países mais pobres, previne novas infecções por pacientes infecciosos e o desenvolvimento de resistência a multidrogas $^{(10)}$.

O TS tem sido abordado sob diferentes aspectos, sob a singularidade do doente ${ }^{(11)}$, sob a ótica da equipe de saú$\mathrm{de}^{(12)}$, sob aspectos organizacionais dentro do sistema de saúde ${ }^{(13-14)}$.

Este estudo tem como objetivo analisar a cobertura do Tratamento Supervisionado (TS) para tuberculose nos municípios prioritários do Estado de São Paulo nos anos de 1998 a 2004. Ele partiu da hipótese de que quanto maior a população de um município, mais complexo o seu sistema de saúde, maior sua capacidade de complementar recursos para as ações de controle da TB e atingir a meta da cobertura do TS que é de $100 \%$, conforme preconiza a OMS.

\section{MÉTODO}

Estudo exploratório que utiliza dados do Banco de Dados EPI-TB da Secretaria Estadual de Saúde de São Paulo registrados até Março de 2006. O estudo incluiu os 36 municípios prioritários para o controle da Tuberculose do Estado de São Paulo, segundo o Plano Nacional de Controle da Tuberculose (1995): Bertioga, Taubaté, Sorocaba, Ubatuba, Suzano, São Caetano do Sul, Carapicuíba, Franco da Rocha, Caraguatatuba, São Vicente, São Sebastião, São Paulo, São Bernardo do Campo, Piracicaba, Osasco, Mogi das Cruzes, Mauá, Diadema, Jacareí, Guarulhos, Bauru, Barueri, Taboão da Serra, São José dos Campos, São José do Rio Preto, Santos, Santo André, Ribeirão Preto, Praia Grande, Jundiaí, Itaquaquecetuba, Cubatão, Embu, Campinas, Guarujá, Itanhaém. Os critérios adotados para a eleição dos municípios prioritários foram: população acima de 50.000 habitantes; coeficiente de incidência acima da média brasileira (58,4 por 100.000 hab.em 1995); óbitos por tuberculose acima de $5 \%$ dos casos novos no ano; cura abaixo de $85 \%$; abandono acima de $10 \%$; índice de sem informação (do resultado de tratamento) acima de 5\%; elevada incidência de casos de $\operatorname{AIDS}^{(3)}$.

Para verificar a cobertura utilizou-se um instrumento de coleta que abordou as seguintes informações: ano de implantação do DOTS nos 36 municípios prioritários, população, casos novos em TS e casos novos sem TS por ano e município no período de 1998 a 2004. $O$ indicador de cobertura do TS foi calculado através da expressão: 
$\mathrm{C}=\mathrm{n}^{\circ}$ de casos novos de pacientes em TS,ano,município

$\times 100 \%$

Total de casos novos, ano, município

\section{ANÁLISE DOS DADOS}

Os dados foram dispostos em uma planilha no Programa Excel utilizando a técnica de dupla verificação e digitação para minimizar possíveis erros na transcrição das informações. Em seguida, realizou-se a construção dos gráficos e análise descritiva dos resultados.

\section{RESULTADOS}

Em relação aos municípios, nove possui população acima de 500 mil habitantes; dezenove entre 200-500 mil habitantes e oito com menos que 200 mil habitantes.

Em 1998, ano da implantação da estratégia DOTS, nos municípios prioritários para o combate da TB do Estado de São Paulo, a cobertura do TS foi inferior a $20 \%$.

Em 1999, observa-se um aumento significativo da cobertura em quase todos os municípios, $18(50 \%)$ entre $11 \%$ e 49\%, 16 (44,44\%) municípios com cobertura menor que $10 \%$, permanecendo apenas $2(11,11 \%)$ com cobertura zero.

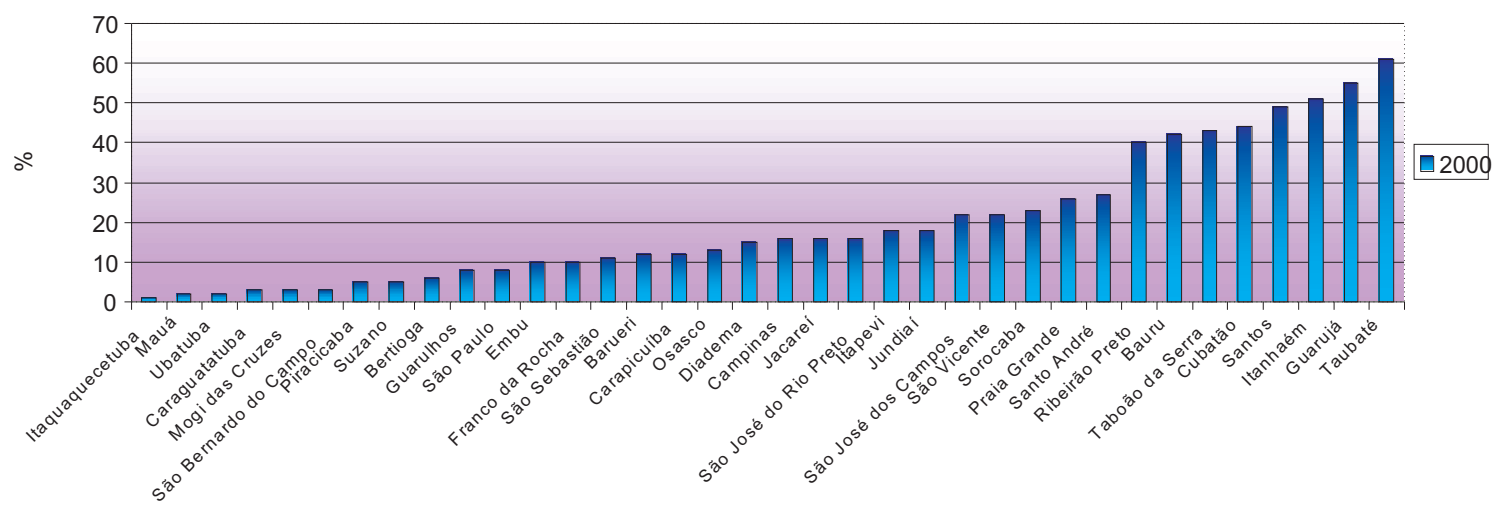

Fonte: SES/EPI-TB, 2006

Figura 1 - Distribuição da cobertura do TS em municípios prioritários no Estado São Paulo - São Paulo - 2000

De acordo com a Figura 1, no ano 2000, todos os 36 (100\%) municípios implantaram o TS, sendo que somente Taubaté, Guarujá e Itanhaém (8,3\% do total) tiveram coberturas acima de $50 \%$. Vinte municípios $(55,56 \%$ do total) tiveram coberturas entre $11 \%$ e $49 \%$ e 13 (36,11\%) dos municípios tiveram menos de $10 \%$. Porém, mesmo com o aumento da cobertura 10 municípios apresenta- ram queda em relação ao ano de 1999, tais como: Caraguatatuba (de 6\% para 3\%), Franco da Rocha (de $15 \%$ para $10 \%$ ), Guarulhos (de $12 \%$ para $8 \%$ ), Jacareí (de $21 \%$ para $16 \%$ ), São Bernardo do Campo (de $4 \%$ para $3 \%$ ), São José do Rio Preto (de $24 \%$ para 16\%), Suzano (de $9 \%$ para $5 \%$ ), Taboão da Serra (de $46 \%$ para $43 \%$ ) e Ubatuba (de 6\% para $2 \%$ ).

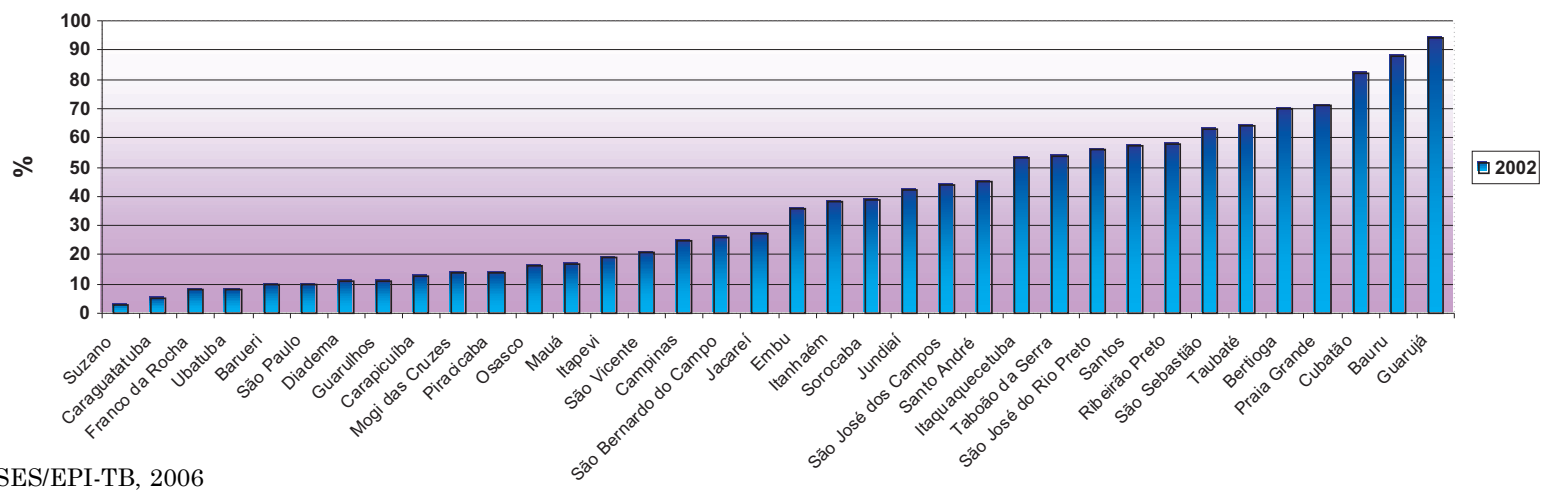

Figura 2 - Distribuição da cobertura do TS em municípios prioritários no Estado São Paulo - São Paulo - 2002

Em 2002, conforme Figura 2 a cobertura aumenta sensivelmente em $25(69,44 \%)$ municípios e decresce em 11 (30,56\%), aumentando de 7 para 11 municípios a cobertura entre 50 e $89 \%$, e dois (2) municípios apresentaram cobertura maior que $90 \%$.
Em 200321 (58,33\%) municípios apresentaram cobertura de TS entre $1 \%$ e $49 \%$ e 14 (38,89\%) maior que $50 \%$, destacando-se Guarujá (95\%) Itanhaém (91\%) e São José dos Campos (82\%). O município de Bertioga, com cobertura de $2 \%$ em 1999 , chegou a $70 \%$ em 2002 e 
apresentou queda brusca em 2003, com cobertura zero, o que indica a necessidade de melhor investigação.

Em 2004, do total de municípios estudados, 2 apresentaram cobertura maior que 90\%, 16 (44,44\%), cobertura entre 50 e $89 \%$. 12 (33,33\%), cobertura entre 10\% e $49 \%$ e apenas $4(11,11 \%)$, cobertura entre $1 \%$ e $10 \%$. Há uma tendência de aumento de cobertura em 23 (63,89\%) municípios.

A Figura 3 descreve a evolução da cobertura do TS nos municípios prioritários para o controle da TB com população entre 101 a 200 mil habitantes.

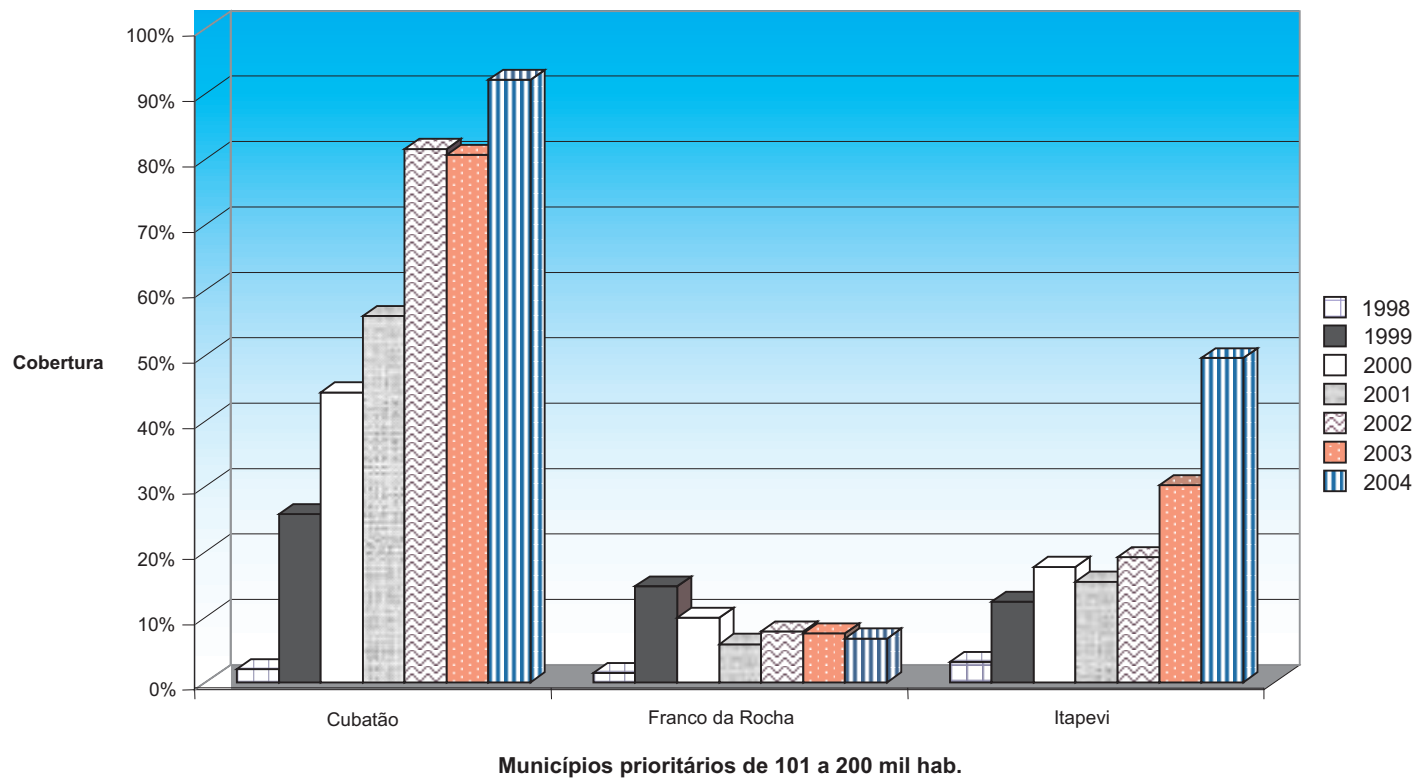

Fonte: SES/EPI-TB, 2006

Figura 3 - Evolução da Cobertura do TS em municípios prioritários no Estado de São Paulo para o controle da TB com população entre 101 a 200 mil habitantes - São Paulo - 1998-2004

Franco da Rocha apesar de ter uma população com menos de 200 mil habitantes apresentou baixa cobertura de TS no período de 1998 a 2004, enquanto que, Cubatão que se encontra na mesma faixa populacional, a cobertura evolui satisfatoriamente estando próximo à meta da OMS, que é de $100 \%$.
A Figura 4 mostra a evolução da cobertura do TS de 1998 a 2004 nos municípios prioritários para o controle da TB com população entre 301 a 400 mil habitantes.

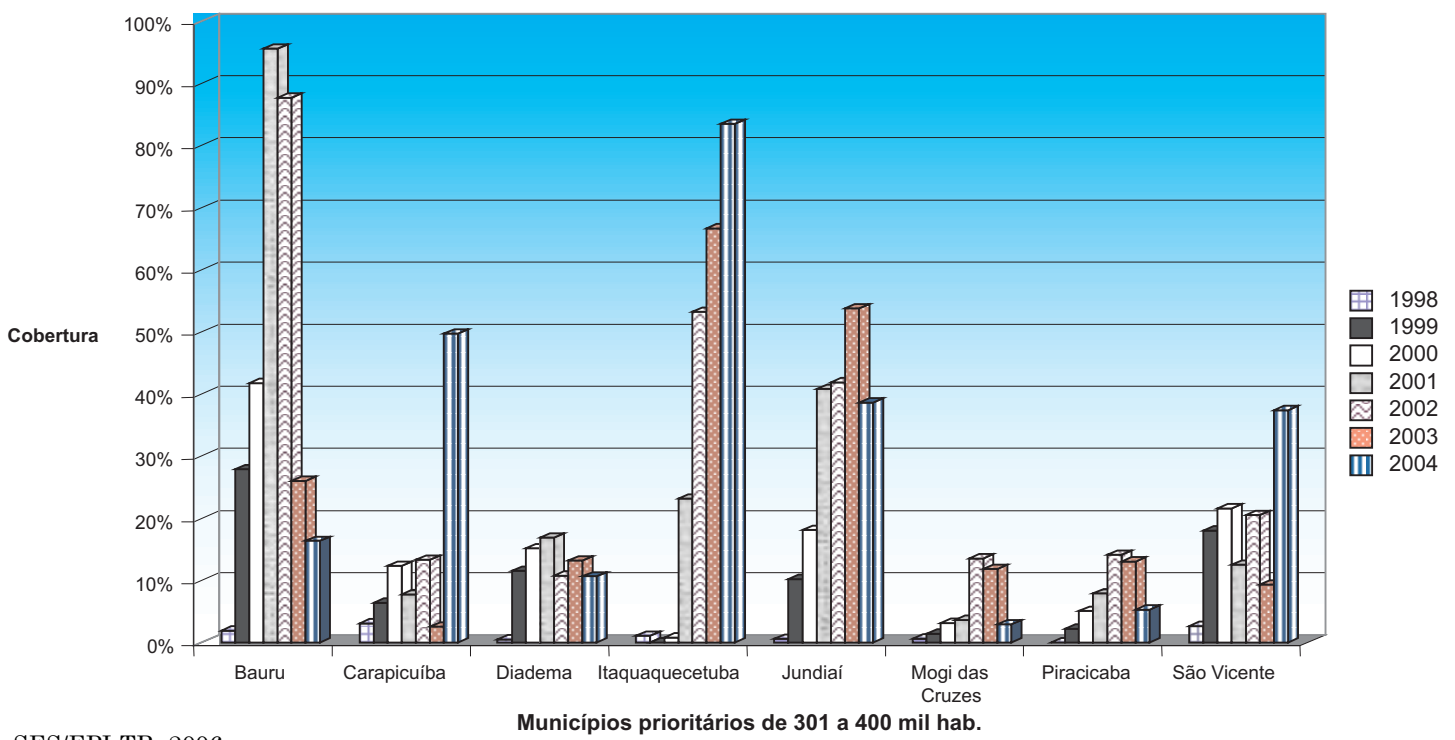

Fonte: SES/EPI-TB, 2006

Figura 4 - Evolução da Cobertura do TS em municípios prioritários no Estado de São Paulo para o controle da TB com população entre 301 a 400 mil habitantes - São Paulo - 1998-2004 
Piracicaba e Itaquaquecetuba, que apresentam números populacionais aproximados (360.762 e 340.596 habitantes respectivamente), não apresentam a mesma evolução na cobertura do TS. Piracicaba não alcança $20 \%$ de cobertura enquanto que Itaquaquecetuba ultrapassa o valor de $80 \%$. O mesmo não acontece com Piracicaba em relação à Mogi das Cruzes ou Diadema pois apresentam semelhantes coberturas de TS e tamanhos populacionais.

A Figura 5 aponta a evolução da cobertura do TS ao longo dos anos em municípios prioritários para o controle da TB com população maior que $600 \mathrm{mil}$ habitantes.

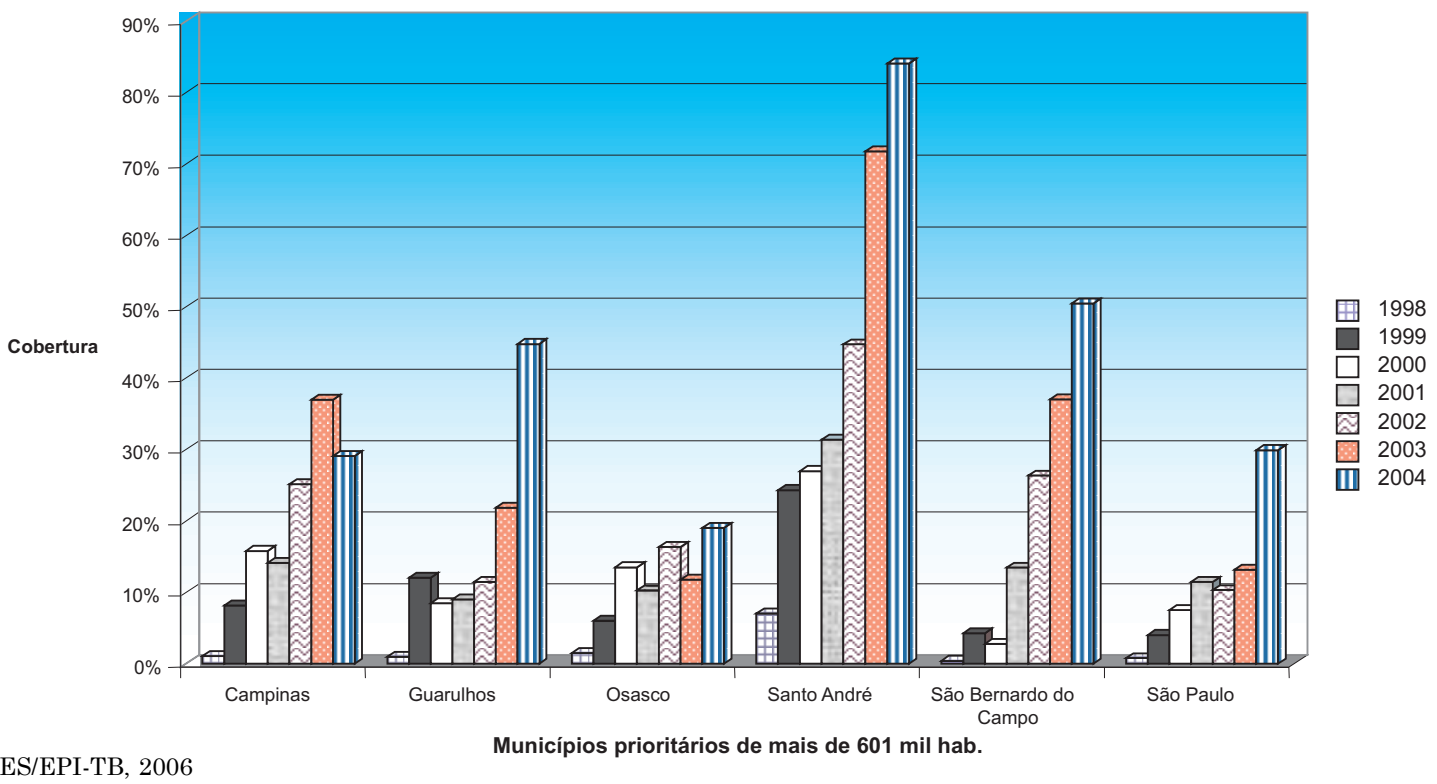

Fonte: SES/EPI-TB, 2006

Figura 5 - Evolução da Cobertura do TS em municípios prioritários no Estado de São Paulo para o controle da TB com população maior que 600 mil habitantes - São Paulo - 1998 - 2004

A evolução da cobertura do TS difere em municípios que estão entre 500 e 1.000 .000 de habitantes, sendo inferior no município de Osasco (705.450 hab.) e superior em Santo André (669.592 hab.). Em Campinas apresentou queda na cobertura entre 2003 e 2004.

\section{DISCUSSÃO}

No período foram traçadas estratégias em âmbito nacional e internacional. No ano 2000, em âmbito internacional, é traçada a Declaração de Amsterdam Stop TB visando acelerar medidas de controle à TB, para alcançar as metas até $2005,70 \%$ de detecção de casos e $85 \%$ de sucesso de tratamento a partir da estratégia DOTS ${ }^{(9)}$.

Em âmbito nacional, a partir de 2001, as ações do PCT passam a ser responsabilidade de todos os municípios, privilegiando as unidades de saúde de nível primário (PSF, UBS, Ambulatórios de Referência) ${ }^{(8,15-16)}$. São estabelecidas metas a serem alcançadas, destacando a proporção de abandono do tratamento da TB como um dos indicadores pactuados $^{(4)}$ e apoiados no Pacto de gestão em defesa do SUS através do fortalecimento da capacidade de respostas às endemias ${ }^{(17)}$.

Os resultados indicam que a Cobertura do TS têm sido crescente no período 1998-2004, porém apresenta oscila- ções na manutenção da cobertura em vários municípios do Estado de São Paulo. Eles mostram que a cobertura do TS não tem relação com o porte do município, uma vez que tem se mostrado heterogênea.

Municípios de regiões metropolitanas como Campinas e Guarulhos, ou seja, de grande porte, por exemplo, apresentaram baixa cobertura de TS em 2004 (29\% e 45\% respectivamente), mostrando que estes enfrentam dificuldades de diferentes ordens as quais podem ser o motivo da baixa cobertura. Já o município de Santo André localiza-se também na região metropolitana, com considerável população, e, no entanto a cobertura do TS alcança $84 \%$ no mesmo ano.

Uma das explicações poderia ser a característica de um município de grande porte organizar seu sistema de saúde em termos de vigilância da TB e monitoramento de casos novos. Outra explicação parte da suposição que, quanto maior o município, mais complexo tende a ser o seu sistema de saúde, e consequentemente, maior sua capacidade de complementar recursos provenientes dos fundos de transferência federal e estadual para a saúde e maior possibilidade de ter autonomia para gestão do sistema municipal de saúde ${ }^{(18)}$.

Houve queda na manutenção da cobertura de TS em vários municípios e as explicações podem envolver a di- 
mensão político-gerencial e de financiamento das ações de saúde (repasse de verbas) a fim de explicar essa falta de sustentabilidade.

A dimensão político-gerencial pode ser explicada pela posição que a TB ocupa na agenda política de saúde do município. Ocorre descontinuidade de cargos de gestor, e/ ou coordenador do PCT, ou o coordenador passa a acumular a coordenação de vários programas ou serviços em decorrência da descentralização para a Atenção Básica(19).

A dimensão do financiamento pode ser explicada pelo grande incentivo às ações de controle da TB, entre 1998 e 2004, a partir da introdução de estratégias como repasse de verbas específicas para o estímulo da busca de casos e o tratamento através de Bônus de $\mathrm{R} \$ 150,00$ [cento e cinqüenta reais] ou $\mathrm{R} \$ 100,00$ [cem reais] se houve ou não Tratamento Supervisionado(20).

\section{CONCLUSÕES}

Conclui-se que houve aumento na cobertura de TS em $63,89 \%$ dos municípios prioritários do Estado de São Paulo (SES) no período estudado, entretanto, alguns municípi- os não conseguiram manter a sustentabilidade da cobertura alcançada.

A hipótese apresentada, de que a Cobertura do TS estaria relacionada ao tamanho populacional e complexidade do sistema de saúde, não foi confirmada, uma vez que os resultados mostram que a cobertura variou em relação ao porte dos municípios tendo se mostrado heterogênea, nem ao fato de ser classificado pela SES/CVE/PCT como município do interior, baixada santista e região metropolitana. Há municípios de populações semelhantes com diferentes coberturas do TS, ou ainda; municípios com populações diferentes e evoluções de cobertura semelhantes. Essa heterogeneidade de cobertura precisa ser estudada buscando a explicação nas dimensões político-gerencial (compromisso político do governo local), técnico-operacional (sistema de saúde) e do financiamento das ações TB.

Esperamos que estas informações geradas sobre a situação da TB possam servir de subsídios para os gestores locais para nova formulação de políticas públicas que incluam o controle da doença de forma efetiva garantindo a sustentabilidade e efetividade destas ações e a melhoria da qualidade dos serviços que prestam assistência aos doentes de TB.

\section{REFERÊNCIAS}

1. Ruffino-Netto A. Programa de Tuberculose no Brasil: situação atual e novas perspectivas. Inf Epidemiol SUS. 2001;10(3):129-38.

2. Ruffino-Netto A. Tuberculose: a calamidade negligenciada. Rev Soc Bras Med Trop. 2002;35(1):51-8.

3. Brasil. Ministério da Saúde. Fundação Nacional de Saúde. Centro Nacional de Epidemiologia. Coordenação Nacional de Pneumologia Sanitária. Programa de Controle da Tuberculose. Diretrizes do plano de ação emergencial para municípios prioritários. Brasília; 1997.

4. Brasil. Ministério da Saúde. Secretaria de Vigilância em Saúde. Departamento de Vigilância Epidemiológica. Coordenação geral de Doenças Endêmicas. Área Técnica de Pneumologia Sanitária. Programa Nacional de Controle da Tuberculose. Brasília; 2004.

5. World Health Organization (WHO). Global tuberculosis control: surveillance, planning, financing. Geneva; 2006. Brazil: country profile; p. 77-9.

6. Instituto Brasileiro de Geografia e Estatística (IBGE). Projeção da população do Brasil, 2004 [texto na Internet]. Rio de Janeiro; 2004. [citado 2006 out. 20]. Disponível em: http://www.ibge.gov.br/cidades

7. Brasil. Ministério da Saúde. DATASUS. Sistema de Informação de Agravos de Notificação [texto na Internet]. Brasília; 2005. [citado 2005 nov. 10]. Disponível em: http: //www.datasus.gov.br.
8. Villa TCS, Monroe AA, Gonzales RIC, Arcêncio RA, Oliveira MFD, Galesi VMN, et al. A experiência de implantação da estratégia DOTS no Estado de São Paulo (1998-2005). In: Ruffino-Netto A, Villa TCS. A implantação do DOTS em algumas regiões do Brasil: histórico e peculiaridades regionais. Ribeirão Preto: Instituto Milênio/Rede TB; 2006. p. 75-139.

9. World Health Organization (WHO). Progress in TB control in high-burden countries, 2001: one year after the Amsterdam Ministerial Conference. Geneva: WHO; 2001.

10. Davies PD. The role of DOTS in tuberculosis treatment. Am J Respir Med. 2003;2(3):203-9.

11. Vendramini SHF, Villa TCS, Palha PF, Monroe AA. Tratamento supervisionado no controle da tuberculose em uma unidade de saúde de Ribeirão Preto: a percepção do doente. Bol Pneumol Sanit. 2002;10(1):5-12.

12. Muniz JN, Villa TCS, Monroe AA, Hino P. Construindo e organizando a prática do Tratamento Supervisionado no controle da Tuberculose. Espaço Saúde [periódico na Internet].2001[citado 2001 jun. 21];2(2):[cerca de 16 p.]. Disponível em: http://www.ccs.uel.br/espaço para saude/v2n2/doc/tuberculose.htm

13. Sassaki CM, Arcêncio RA, Costa Junior ML, Palha PF, Cardozo-Gonzales RI, Villa TCS. Tempo de tratamento de pacientes inscritos no Programa de Controle da Tuberculose. Ribeirão Preto- SP (1998-1999). Bol Pneumol Sanit. 2002;10(2):27-34. 
14. Vendramini SHF, Villa TCS, Cardozo-Gonzales RI, Monroe AA. Tuberculose no idoso: análise do conceito. Rev Lat Am Enferm. 2003;11(1):96-103.

15. Brasil. Ministério da Saúde. Portaria n. 373, de 27 de fevereiro de 2002. Norma Operacional da Assistência à Saúde - NOAS SUS 01/2002 [legislação na Internet]. Brasília; 2002. [citado 2005 fev. 15]. Disponível em: http://www.ccs.uel.br/nesco/regesus/arquivos/noas 01 2002.pdf

16. Brasil. Ministério da Saúde. Secretaria das Políticas de Saúde. Departamento de Atenção Básica. Coordenação Nacional de Pneumologia Sanitária. Plano de Controle da Tuberculose no Brasil no período de 2001-2005. Brasília; 2000.

17. Brasil. Ministério da Saúde. Organização Pan Americana de Saúde (OPAS). Desenvolvimento de Sistemas de Serviços de Saúde. Validação de uma metodologia de avaliação rápida das características organizacionais e do desempenho dos serviços de atenção básica do Sistema Único de Saúde (SUS) em nível local. Brasília; 2006.
18. Cohn A, Westphal MF, Elias PE. Informação e decisão política em saúde. Rev Saúde Pública. 2005;39(1): 114-21.

19. Muniz JN, Ruffino-Netto A, Villa TCS, Yamamura M, Arcêncio RA, Cardozo-Gonzales RI. Aspectos epidemiológicos da co-infecção tuberculose - HIV em Ribeirão Preto - SP (1998-2003). J Bras Pneumol. 2006; 32(6):529-34.

20. Villa TCS, Ruffino-Netto A, Arcêncio RA, Cardozo-Gonzales RI. As Políticas de Controle da Tuberculose no Sistema de Saúde no Brasil e a implantação da Estratégia DOTS (1980-2005). In: Ruffino-Netto A, Villa TCS A implantação do DOTS em algumas regiões do Brasil: histórico e peculiaridades regionais. Ribeirão Preto: Instituto Milênio/Rede TB; 2006. p. 29-47.

\section{Agradecimentos}

Projeto Temático FAPESP 2004-2007 processo 03/08386-3, Rede Brasileira de Pesquisas em Tuberculose - REDE-TB e à Secretaria Estadual de Saúde de São Paulo. 